\title{
Whole-Station Visualization of Secondary Circuit in Smart Substation Based on Hybrid Topology
}

\author{
Chao Sun ${ }^{1}$, Xintong Liu $^{1 *}$, Zhaowu Yin ${ }^{2}$, Yingjie Di $^{2}$, Zhiyong Wang ${ }^{2}$ \\ ${ }^{1}$ Hebei University of Water Resources and Electric Engineering, Cangzhou 061001, China \\ ${ }^{2}$ State Grid Jibei Electric Power Company Limited Laoting Power Supply Company, Tangshan 063600, China
}

Corresponding Author Email: liuxintong@hbwe.edu.cn

https://doi.org/10.18280/ejee.220210

Received: 12 November 2019

Accepted: 17 February 2020

\section{Keywords:}

smart substation, secondary circuit, visualization, hybrid topology

\begin{abstract}
For smart substations, the available visualization tools cannot display all the information of the secondary circuit, which hinders the field work of operation and maintenance personnel. To solve the problem, this paper fully analyzes the virtual connections between protection devices and intelligent electronic devices (IEDs) in smart substation, and develops a whole-station visualization method of secondary circuit in smart substation, drawing on hybrid topology. The visualization process was introduced in details, including key steps like the judgment of core IEDs, generation of local diagrams, and the formulation of the whole-station secondary circuit diagram. The proposed method was further explained with an example. The example analysis shows that our method could visually display the connections and data transmission between secondary devices in smart substation, allowing relevant personnel to quickly familiarize with the network structure and virtual connections of secondary devices throughout the substation. The research results help to reduce omissions and incorrectness in operations, and push up the operational efficiency in smart substations.
\end{abstract}

\section{INTRODUCTION}

Smart substation differs greatly from conventional substation in secondary circuit: the cable is replaced by optical fiber, and the physical secondary terminal block is replaced by a virtual terminal list. In other words, the physical connections in the secondary circuit are changed into the virtual connections between intelligent electronic devices (IEDs) in substation configuration description (SCD) [1-5]. In engineering, virtual terminals refer to the model information of the IEDs, as well as their connections. The importance of virtual terminals, which are related to the connections of secondary circuit, are self-evident. In the field of smart substation, much attention has been paid to the modeling, automatic connection, visualization, and detection of virtual terminals. Among them, the visualization of virtual terminals is a subject in need of further research.

Most power operation and maintenance personnel are used to working with drawings like terminal block diagram. It is difficult for them to inspect, debug, and maintain virtual terminals, which are invisible and untouchable. To overcome the difficulty, graphic visualization should be conducted to visually display the device models and virtual connections in the SCD, creating a graph with a good spatial layout and high similarity to the conventional secondary circuit diagram. The graph will provide a good reference for project acceptance, circuit check, fault handling, and technical training, and thus improve the work efficiency of power operation and maintenance personnel [6-8].

Currently, there are many studies on the visualization of the secondary circuit in smart substation. Based on the information flows from different angles, some scholars [9-13] proposed graphical generation methods and solutions of SCD virtual terminals. Focusing on SCD comparison, some scholars [14-16] visually processed the SCD to enhance the readability of information, and improve the efficiency of power operation and maintenance personnel. Some scholars [17-20] provided technical solutions for visual operation and maintenance based on different models, and verified them through engineering application. Overall, the existing research results focus on the visualization of single equipment and its associated equipment, as well as the relevant techniques like testing and safety precautions. There is not yet any report on the visualization of the secondary circuit of the whole smart substation.

This paper mainly deals with the visualization of secondary circuit in smart substation. The secondary circuit configuration diagram of the whole station was generated dynamically, providing an innovative graphical display of the virtual connections between the protection devices and intelligent components. The visual display of whole-station virtual connections greatly facilitates the equipment operation by designers, as well as power operation and maintenance personnel. The research results have great significance in theory and practice.

Based on hybrid topology, this paper presents a method to display the secondary circuit of the entire smart substation. The visualization process was introduced in details, including key steps like the judgment of core IEDs, generation of local diagrams, and the formulation of the whole-station secondary circuit diagram. The proposed method was further explained with an example. 


\section{STATUS QUO OF SECONDARY CIRCUIT VISUALIZATION}

In smart substation, the information of secondary circuit is stored such files as virtual terminal list, equipment network diagram, the SCD, etc. These files are inconvenient to query or view, and should be visualized. Currently, secondary circuit is usually visualized into the following forms: virtual terminal diagram, secondary topology diagram, logical link diagram, main transformer association diagram, and bus association diagram.

Specifically, virtual terminal diagram displays the information of all leading-in and leading-out virtual terminals in each device of the process layer, enabling users to view device information easily. This diagram is of great help to device renovation and substation expansion. Like the circuit diagram of conventional substation, secondary topology diagram presents the virtual terminal connections between each device and other devices. Logical link diagram shows the logical links between each device and other devices; the logical links, described with network parameters, demonstrate the connections between specific information. The main transformer association diagram and bus association diagram display the devices that protect the main transformer and the bus, respectively [21-23].

The existing secondary circuit visualization tools roughly fall into two categories, namely, system integration tools and digital instrument tools. The former tools provide an IED dataset and virtual terminal connections, and focus on the configuration of smart substation. The latter tools can graphically display the connections between virtual terminals, but fail to illustrate the entire model dataset or communication settings of the IEDs [24-26]. To sum up, each type of tools has its merits and defects. Neither type of tools is comprehensive enough in function. Further integration and improvement are necessary to develop between visualization tools for secondary circuit in smart substation.

\section{WHOLE-STATION VISUALIZATION STRATEGY FOR SECONDARY CIRCUIT OF SMART SUBSTATION}

Drawing on hybrid topology, the visualization strategy for whole-station secondary circuit of smart substation was determined through the analysis on the SCD of smart substation:

(1) Selection of core devices: The SCD file should be analyzed to identify the important protection devices in the substation and their virtual connections.

(2) Construction of relationship matrix: The virtual connections between core devices should be described as a matrix.

(3) Definition of drawing priority: The sequence of core devices in drawing process should be determined based on the values in the relationship matrix.

(4) Generation of local diagrams: Every protection device (drawing priority $=1$ ) should be taken as a central node, while the relevant IEDs as secondary nodes. Based on relationship matrix and drawing priorities, the local diagrams should be generated based on the core devices.

(5) Generation of whole-station diagram: The local diagrams, which correspond to different core devices, should be merged dynamically into the whole-station secondary circuit diagram.

Each of the above key technique will be explained in details in the following section.

\section{KEY TECHNIQUES FOR WHOLE-STATION VISUALIZATION OF SECONDARY CIRCUIT IN SMART SUBSTATION}

\subsection{Selection and preliminary classification of core devices}

For simplicity, the devices in smart substation were classified preliminarily, so that the core devices could be prioritized in the drawing process.

After analyzing the core devices in smart substation and their protection principles, the key IEDs in the secondary circuit were divided into main transformer protection devices, merging units, intelligent terminals, and automatic backup switching devices. These devices are referred to as core devices in this research. The complex correlations between these devices may lead to omissions or incorrectness in maintenance and defect elimination. Special attention should be paid to prepare targeted control measures for such devices.

According to the serial number or description of the IEDs, the core devices were selected from the device list in the SCD file, and classified in a preliminary manner. The classification aims to roughly delineate the area of each device in the topology graph. The devices in the smart station were divided into three categories:

Class 1 devices: The core devices whose description contains "transformer protection", "protection", or "PT", or the core devices whose serial number contains "PT".

Class 2 devices: The core devices whose description contains "automatic backup switch", "backup switch", or "merging unit", or the core devices whose serial number contains "PZ" or "MM".

Class 3: The core devices not classified into Classes 1 and 2

\subsection{Construction of relationship matrix}

The SCD analysis shows that the virtual connections are too complex to visually reflect the relationship between the selected core devices. Hence, the virtual connections between core devices were formulated into a matrix, such that the devices could be further classified based on the connection rules.

According to the preliminary classification, the core devices were sorted out as three column vectors $C_{1}, C_{2}$, and $C_{3}$, which represent the lists of Classes 1-3 devices, respectively. The three column vectors were used to set up a matrix $C$ :

$$
C_{m \times m}=\left(\begin{array}{l}
C_{1} \\
C_{2} \\
C_{3}
\end{array}\right)\left(C_{1}^{T} C_{2}^{T} C_{3}^{T}\right)=\left(\begin{array}{lll}
C_{11} & C_{12} & C_{13} \\
C_{21} & C_{22} & C_{23} \\
C_{31} & C_{32} & C_{33}
\end{array}\right)=\left(\begin{array}{lllll}
c_{11} & c_{12} & \cdots & \cdots & c_{1 m} \\
& \ddots & & & c_{2 m} \\
& & \ddots & & \vdots \\
& & & \ddots & \vdots \\
C_{m 1} & & & & C_{m m}
\end{array}\right)
$$

If $C_{i j}=1$, device i sends GOOSE or SV signal to device j; If $C_{i j}=0$, device $\mathrm{i}$ is not correlated with device $\mathrm{j}$.

Furthermore, matrix $C$ was tripled into a new matrix $D$, where the values in the lower left part were zeroed. The new matrix represents the virtual connections between core device in smart substation: 


$$
D=C+2 C^{T}=\left(\begin{array}{lll}
D_{11} & D_{12} & D_{13} \\
& D_{22} & D_{23} \\
& & D_{33}
\end{array}\right)=\left[\begin{array}{ccccc}
d_{11} & d_{12} & \cdots & \cdots & d_{1 m} \\
0 & \ddots & & & d_{2 m} \\
\vdots & \ddots & \ddots & & \vdots \\
\vdots & & \ddots & \ddots & \vdots \\
0 & \cdots & \cdots & 0 & d_{m m}
\end{array}\right]
$$

where, $d_{i j}=1$ means device $\mathrm{i}$ sends GOOSE or SV signal to device $j(i<j) ; d_{i j}=2$ means device $\mathrm{j}$ sends GOOSE or SV signal to device $\mathrm{i}(i<j) ; d_{i j}=3$ means devices $\mathrm{i}$ and $\mathrm{j}$ send GOOSE or $\mathrm{SV}$ signal to each other $(i<j) ; d_{i j}=0$ means device $\mathrm{i}$ is not correlated with device $\mathrm{j}$.

\subsection{Definition of drawing priority}

The drawing priority refers to the order that devices are placed in the visual graph. The smaller the priority, the more complex the information flow of the device, and the earlier the device appears in the graph. For each core device, the drawing priority was determined based on the preliminary class and relationship matrix $D$. Table 1 lists the preliminary classes and drawing priorities of each type of devices.

Table 1. The preliminary classes and drawing priorities of each type of devices

\begin{tabular}{|c|c|c|}
\hline $\begin{array}{c}\text { Preliminary } \\
\text { classes }\end{array}$ & $\begin{array}{l}\text { Drawing } \\
\text { priorities }\end{array}$ & $\begin{array}{c}\text { Definition rules for drawing } \\
\text { priorities }\end{array}$ \\
\hline 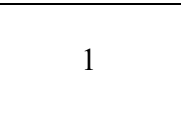 & 然 & $\begin{array}{l}\text { The core devices whose information } \\
\text { contains "transformer protection", } \\
\text { "protection", or "PT". }\end{array}$ \\
\hline 2 & 2 & $\begin{array}{l}\text { The core devices whose information } \\
\text { contains "merging unit", "automatic } \\
\text { backup switch", "backup switch", } \\
\text { "PZ", or "MM". }\end{array}$ \\
\hline \multirow{4}{*}{3} & 31 & $\begin{array}{c}\text { The core devices correlated with } \\
\text { two Class } 1 \text { devices and multiple } \\
\text { Class } 2 \text { devices. }\end{array}$ \\
\hline & 32 & $\begin{array}{c}\text { The core devices correlated with one } \\
\text { Class } 1 \text { device and several Class } 2 \\
\text { devices. }\end{array}$ \\
\hline & 33 & $\begin{array}{c}\text { The core devices only correlated } \\
\text { with one Class } 1 \text { device. }\end{array}$ \\
\hline & 34 & $\begin{array}{l}\text { The core device not correlated with } \\
\text { Class } 1 \text { devices. }\end{array}$ \\
\hline
\end{tabular}

Based on relationship matrix $D$, the further classification of core devices aims to determine the sequence (priority) of each device in the drawing process, and the location of each device in the whole diagram. The specific rules for determining the drawing priority are as follows:

(1) Class 1 devices

The drawing priority is 1 for each Class 1 device.

(2) Class 2 devices

For any Class 2 device, the row $i$ of the device in the relationship matrix was identified. Then, the columns $j$ of the nonzero elements in the row were found out. After that, the devices correlated with that device were determined based on the elements in these columns:

The drawing priority is 32 for each Class 2 device correlated with one Class 1 device and one Class 2 device.

The drawing priority is 33 for each Class 2 device correlated with only one Class 1 device.

The drawing priority is 2 for each Class 2 device in other conditions.

(3) Class 3 devices
For any Class 3 device, the row $i$ of the device in the relationship matrix was identified. Then, the columns $j$ of the nonzero elements in the row were found out. After that, the devices correlated with that device were determined based on the elements in these columns:

The drawing priority is 31 for each Class 3 device correlated with two Class 1 devices.

The drawing priority is 32 for each Class 3 device correlated with one Class 1 device, and multiple Class 2 devices.

The drawing priority is 33 for each Class 3 device correlated with only one Class 1 device.

The drawing priority is 34 for each Class 3 device in other conditions.

\subsection{Diagram generation based on hybrid topology}

A hybrid topology integrates the merits of two single topologies. The commonly used hybrid topologies are starring topology and star-bus topology. As their names suggests, the star-ring topology combines star topology and ring topology, while star-ring topology combines star topology and bus topology. In this paper, the star-bus topology is adopted to visually represent the IEDs in smart substation and their logical links. The whole-station secondary circuit diagram was merged from the star-shaped local diagrams and the ringshaped protection device association diagram. The diagram generation process is described as follows:

Step 1. Take a protection device (core device with drawing priority of 1) as the central node, identify the core devices correlated with this device, and form a star-shaped local diagram.

Step 2. Dynamically integrate the virtual connections between devices with drawing priorities of 1 and 31 into a ring-shaped diagram.

Step 3. Determine the location of each local diagram in the ring-shaped diagram, according to the virtual information, and place the local diagrams at the corresponding locations.

Suppose there are 3 protection devices in the entire substation, denoted as $\mathrm{A}, \mathrm{B}$, and $\mathrm{C}$, respectively. The core devices correlated with both A and B, correlated with both $\mathrm{B}$ and $\mathrm{C}$, and correlated with both $\mathrm{A}$ and $\mathrm{C}$, are denoted as $\mathrm{a}, \mathrm{b}$, and $\mathrm{c}$, respectively. Then, device a should be place between the local diagram of $\mathrm{A}$ and that of $\mathrm{B}$; device $\mathrm{b}$ should be placed between the local diagram of $\mathrm{B}$ and that of $\mathrm{C}$.

If $\mathrm{A}$ is placed in the upper left corner, then $\mathrm{B}$ could be placed in the upper right corner, and $\mathrm{C}$ below $\mathrm{A}$ and $\mathrm{B}$. In this way, the local diagrams of $\mathrm{A}, \mathrm{B}$, and $\mathrm{C}$ could be placed reasonably in the ring-shaped topology.

Step 4. Merge the local diagrams with the same name of core device into one diagram, without changing the correlations between the device and other devices.

Suppose the local diagram of A contains IED a, which is connected with IED $\mathrm{b}$; the local diagram of $\mathrm{B}$ also contains IED a. Then, the two IED as must be merged, and the merged IED should still be correlated with $\mathrm{A}, \mathrm{b}$, and $\mathrm{B}$.

\section{EXAMPLE ANALYSIS}

To verify its effectiveness, the proposed whole-station visualization strategy for secondary circuit was applied to Jingkai substation, a $110 \mathrm{kV}$ smart substation with two voltage levels and 39 IEDs. 
First, the core devices to be displayed were selected by traversing the SCD file. Based on the selection rules, a total of 22 core devices and their virtual correlations were selected, including 4 protection devices and 18 IEDs. Hence, 22 devices should be displayed on the whole-station secondary circuit diagram.
Next, the relationship matrix $D_{22 \times 22}$ (Table 2) was set up based on the preliminary classes and virtual connections of the selected core devices. In the matrix, the value of each element clearly represents the virtual connection and signal propagation direction between devices.

Table 2. Part of the relationship matrix between core devices

\begin{tabular}{ccccccccc}
\hline & PT1101A & PT1101B & PT1102A & PT1102B & PZ1101 & PZ1001 & MM1101 & MM1102 \\
\hline PT1101A & 0 & 0 & 0 & 0 & $\mathbf{1}$ & $\mathbf{1}$ & $\mathbf{0}$ & $\mathbf{0}$ \\
PT1101B & 0 & 0 & 0 & 0 & $\mathbf{1}$ & $\mathbf{1}$ & $\mathbf{0}$ & $\mathbf{0}$ \\
PT1102A & 0 & 0 & 0 & 0 & $\mathbf{1}$ & $\mathbf{1}$ & $\mathbf{0}$ & $\mathbf{0}$ \\
PT1102B & 0 & 0 & 0 & 0 & $\mathbf{1}$ & $\mathbf{1}$ & $\mathbf{0}$ & $\mathbf{0}$ \\
PZ1101 & 0 & 0 & 0 & 0 & 0 & 0 & 0 & 0 \\
PZ1001 & 0 & 0 & 0 & 0 & 0 & 0 & 0 & 0 \\
MM1101 & 0 & 0 & 0 & 0 & 0 & 0 & 0 & 0 \\
MM1102 & 0 & 0 & 0 & 0 & 0 & 0 & 0 & 0 \\
\hline
\end{tabular}

Submatrix $D_{1,2}$ shows the virtual connections between Classes 1 and 2 devices. Taking PT1101A for example, $d_{l, 5}$ and $d_{1,6}=1$ in the first row, indicating that PZ1101 and PZ1001 are information senders.

Submatrix $D_{1,3}$ shows the virtual connections between Classes 1 and 3 devices. Take PT1101A for example, the elements in the first row indicate that the device is virtually correlated with IT1101, IE1101A, IT1001A, and IL1101A. Since $d_{l, 9}=1$, IT1101 is an information sender. Since $d_{l, 10}, d_{l, 13}$ and $d_{1,14}=3$, IT1001A, IL1101A, and IE1101 both send and receive information.

Then, the drawing priorities of Classes 2 and 3 devices were determined based on the relationship matrix D. For Class 2 devices, the definition of drawing priority was explained with MM1101 as an example. This device was found to appear in row 7 of the relationship matrix. In this row, there are $d_{7,10}=2$, $d_{7,11}=1, d_{7,17}=1$, and $d_{7,18}=1$, indicating that MM1101 is virtually correlated with IE1101A, IL1101A, IL1102A, and IT1002A. Hence, MM1101 is correlated with four Class 3 devices, and was thus assigned the drawing priority of 2 . All Class 2 devices were traversed as above to determine their drawing priorities.

For Class 3 devices, the definition of drawing priority was explained with IL1101A as an example. This device was found to appear in row 14 of the relationship matrix. In this row, there are $d_{14,1}=3, d_{14,5}=1$, and $d_{14,7}=2$, indicating that IL1101A is virtually correlated with PT1101A, PZ1101, and MM1101. Hence, IL1101A is correlated with one Class 1 device and two Class 2 devices, and was thus assigned the drawing priority of 32. All Class 3 devices were traversed as above to determine their drawing priorities.

After that, star-shaped local diagrams were prepared with protection devices as central nodes. Take PT1101A (drawing priority $=1$ ) for example. This device was virtually correlated with 6 devices, including two with drawing priority of 32 (IT1001A and IL1101A), two with drawing priority of 2 (PZ1001 and PZ1101), and two with drawing priority of 31 (IE1101A and IT1101). The star-shaped local diagram centering on PT1101A can be organized as Figure 1 below.

Then, a ring-shaped topology was developed based on the virtual connections between devices with drawing priority of 1 , and those with drawing priority of 31 . The smart substation includes 4 devices with drawing priority of 1 , and 4 with drawing priority of 31 . The ring-shaped topology thus obtained is shown in Figure 2.
Based on the virtual connections, the locations of the four local diagrams in the ring diagram were identified. Then, the four local diagrams were placed at these locations. The local diagrams with the same name of core device were merged into one diagram, without changing the correlations between the device and other devices. According to the relationship matrix, the arrow represents the propagation direction of GOOSE and SV signals. In this way, the secondary circuit diagram of Jingkai substation was completed (Figure 3).

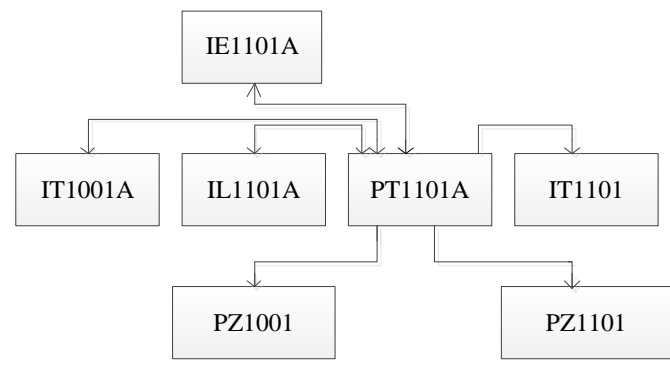

Figure 1. The star-shaped local diagram centering on PT1101A

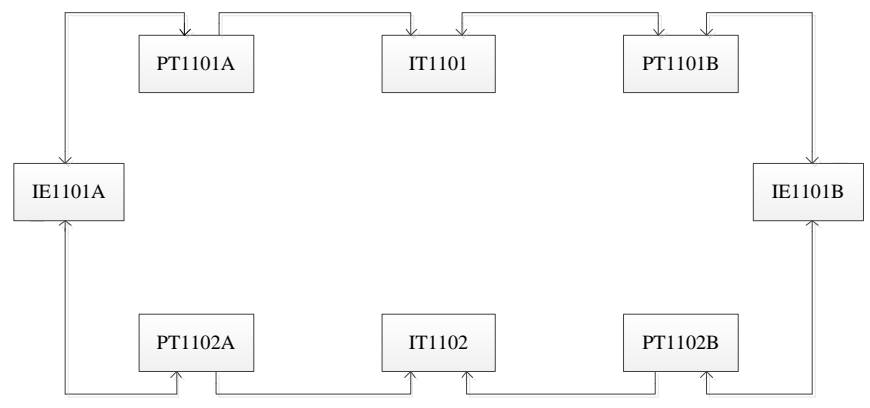

Figure 2. The ring-shaped topology

\section{CONCLUSIONS}

The current visualization methods for secondary circuit in smart substation only display the virtual connections or logical links between a single device and other devices, failing to present the secondary circuit of the entire substation. To make up for the gap, this paper puts forward a novel whole-station visualization method for secondary circuit based on hybrid topology. The core devices were selected to filter out the 
unimportant devices, reducing the complexity of the wholestation diagram. The relationship matrix was established to sort out the virtual connections between IEDs, which simplifies the queries. Besides, the spatial layout of devices was optimized, drawing on hybrid topology and drawing priority. During the diagram generation, local diagrams centering on protection devices were prepared first, and then dynamically merged into the whole-station secondary circuit diagram, according to the relationship between protection devices.

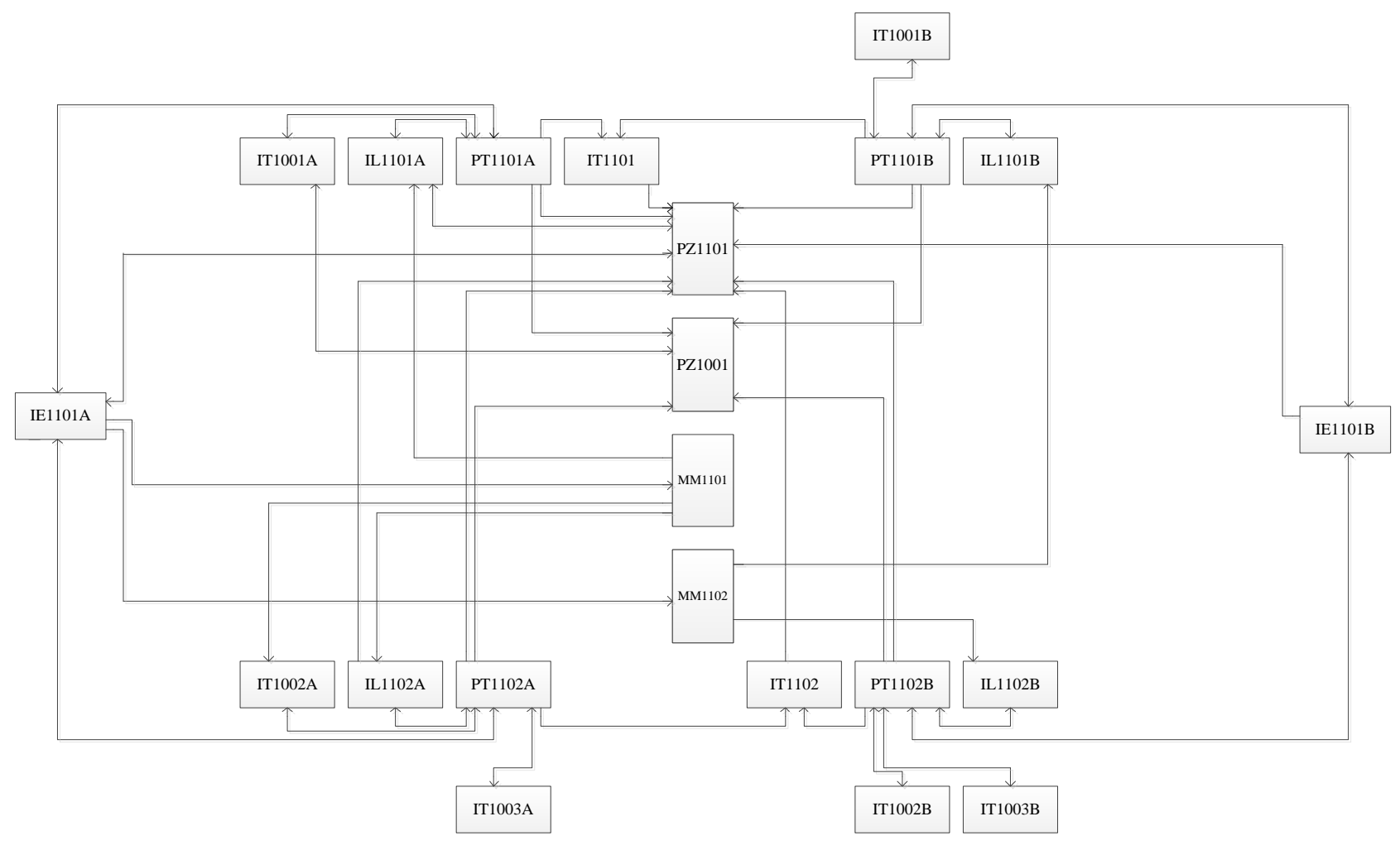

Figure 3. Secondary circuit diagram of Jingkai substation

The engineering example shows our visualization method provides an innovative graphical display of the virtual connections between protection devices and IEDs in the entire substation. There are multiple advantages of our method: The output diagram covers lots of devices, boasts a large information capacity, and clarifies the structure of the virtual connections, allowing relevant personnel to quickly familiarize with the network structure and virtual connections of secondary devices throughout the substation. Besides, the whole-station secondary circuit diagram is highly scalable. Taking the diagram as an information container, various information could be added, namely, soft pressure plates, network communication parameters, and switch models, which helps to improve the efficiency of professional jobs like design, debugging, operation and maintenance.

\section{ACKNOWLEDGEMENT}

This work was supported by the Science and Technology Planning Project of Hebei Province (self-funded) under Grant No.18212108 and the Science and Technology Guidance Project of Cangzhou under Grant No. 172104002.

\section{REFERENCES}

[1] Heckel, J. (2009) Smart substation and feeder automation for a SMART distribution grid. (2009). CIRED 2009 20th International Conference and Exhibition on
Electricity Distribution - Part 1, Prague, Czech Republic, pp. 1-4. https://doi.org/10.1049/cp.2009.0942

[2] Aftab, M.A., Hussain, S.S., Ali, I., Ustun, T.S. (2020). IEC 61850 based substation automation system: A survey. International Journal of Electrical Power \& Energy $\quad$ Systems, 120: 106008. https://doi.org/10.1016/j.ijepes.2020.106008

[3] Huang, Q., Jing, S., Li, J., Cai, D., Wu, J., Zhen, W. (2017). Smart substation: state of the art and future development. IEEE Transactions on Power Delivery, 32(2): 1098-1105. https://doi.org/10.1109/TPWRD.2016.2523520

[4] Leonardi, A., Mathioudakis, K., Wiesmaier, A., Zeiger, F. (2014). Towards the smart grid: substation automation architecture and technologies. Advances in Electrical Engineering, 2014: https://doi.org/10.1155/2014/896296

[5] Kim, H., Kim, J.K., Song, J., Lee, J., Hur, K. (2019). Smart and green substation: shaping the electric power grid of Korea. IEEE Power and Energy Magazine, 17(4): 24-34. https://doi.org/10.1109/MPE.2019.2909792

[6] Gomez-Quiles, C., Gomez-Exposito, A., de la Villa Jaen, A. (2012). State estimation for smart distribution substations. IEEE Transactions on Smart Grid, 3(2): 986995. https://doi.org/10.1109/tsg.2012.2189140

[7] Ali, N.H., Ali, B.M., Basir, O., Othman, M.L., Hashim, F., Abdel-Latif, K.M. (2016). Protection of smart substation based on WLAN complies with IEC 61850 using traveling wave analysis. Electric Power Systems Research, $140(11)$ 
https://doi.org/10.1016/j.epsr.2016.07.004

[8] Khuntia, S.R., Rueda, J.L., Bouwman, S., van der Meijden, M.A. (2016). A literature survey on asset management in electrical power [transmission and distribution] system. International Transactions on Electrical Energy Systems, 26(10): 2123-2133. https://doi.org/10.1002/etep.2193

[9] Gu, J.C., Liu, C.H., Wang, J.M., Yang, M.T. (2019). Using IEC 61850 GOOSE messages in microgrid protection. International Transactions on Electrical Energy Systems, 29(12): e12122. https://doi.org/10.1002/2050-7038.12122

[10] Mohamed, H. (2018). Goose performance assessment on an IEC 61850 redundant network. The Journal of Engineering, 2018(15): 841-845. https://doi.org/10.1049/joe.2018.0208

[11] Hussain, S.M.S., Ustun, T.S., Kalam, A. (2019). A review of IEC 62351 security mechanisms for IEC 61850 message exchanges. IEEE Transactions on Industrial Informatics. https://doi.org/10.1109/TII.2019.2956734

[12] Jing, S., Huang, Q., Wu, J., Zhen, W. (2013). A novel whole-view test approach for onsite commissioning in smart substation. IEEE Transactions on Power Delivery, 28(3):

1715-1722. https://doi.org/10.1109/tpwrd.2013.2256435

[13] Haidong Zhang, Renhui Dou, Mingzhi Geng, \& Qing Yang. (2016). The technology of interlocking logic visualization for smart substation. 2016 China International Conference on Electricity Distribution (CICED), Xi'an, pp. 1-4. https://doi.org/10.1109/CICED.2016.7575944

[14] Aftab, M.A., Hussain, S.M.S., Ali, I., Ustun, T.S. (2019). A novel SCL configuration method for modeling microgrids with IEC 61850. IEEE Systems Journal. https://doi.org/10.1109/JSYST.2019.2917927

[15] Nadeem, F., Aftab, M.A., Hussain, S.M., Ali, I., Tiwari, P.K., Goswami, A.K., Ustun, T.S. (2019). Virtual power plant management in smart grids with XMPP based IEC 61850 communication. Energies, 12(12): 2398. https://doi.org/10.3390/en12122398

[16] Jing, S., Huang, Q., Wu, J., Zhen, W. (2013). A novel whole-view test approach for onsite commissioning in smart substation. IEEE Transactions on Power Delivery, 28(3):

$1715-1722$. https://doi.org/10.1109/tpwrd.2013.2256435

[17] Zhao, G.Q., Fan, C., Yuan, H., Ren, H. (2016). The research on the integrated test system for the secondary devices and system in smart substation. 2016 IEEE PES Asia-Pacific Power and Energy Engineering Conference (APPEEC), Xi'an, pp. 1817-1821. https://doi.org/10.1109/APPEEC.2016.7779803

[18] Hussain, A., Kim, H.M. (2016). A hybrid framework for adaptive protection of microgrids based on IEC 61850 . International Journal of Smart Home, 10(5): 285-296. https://doi.org/10.14257/ijsh.2016.10.5.26

[19] Lei, H., Singh, C., Sprintson, A. (2014). Reliability modeling and analysis of IEC 61850 based substation protection systems. Smart Grid IEEE Transactions on, $5(5)$ :

2194-2202. https://doi.org/10.1109/tsg.2014.2314616

[20] Ustun, T.S. (2016). Interoperability and interchangeability for microgrid protection systems using IEC 61850 standard. 2016 IEEE International Conference on Power and Energy (PECon), Melaka, pp. 7-12. https://doi.org/10.1109/PECON.2016.7951463

[21] Matta, N., Rahim-Amoud, R., Merghem-Boulahia, L., Jrad, A. (2018). Putting sensor data to the service of the smart grid: from the substation to the AMI. Journal of Network and Systems Management, 26(1): 108-126. https://doi.org/10.1007/s10922-017-9409-0

[22] Leal, A., Botero, J.F. (2019). Defining a reliable network topology in software-defined power substations. IEEE Access, 7: 14323-14339. https://doi.org/10.1109/ACCESS.2019.2893114

[23] Kumar, S., Islam, S., Das, N., Gardner, B. (2016). Notice of removal NCIT enabled OPNET based design of a digital substation for IEC 61850-9-2 implementation. IEEE Transactions on Power Delivery. https://doi.org/10.1109/TPWRD.2016.2524670

[24] Brahma, S. (2016). Advancements in centralized protection and control within a substation. IEEE Transactions on Power Delivery, 31(4): 1945-1952. https://doi.org/10.1109/TPWRD.2016.2528958

[25] Ferreira, V.H., Zanghi, R., Fortes, M.Z., Sotelo, G.G., Silva, R.B.M., Souza, J.C.S., Guimarães, C.H.C., Gomes Jr, S. (2016). A survey on intelligent system application to fault diagnosis in electric power system transmission lines. Electric Power Systems Research, 136: 135-153. https://doi.org/10.1016/j.epsr.2016.02.002

[26] Lim, I.H., Sidhu, T.S. (2013). Design of a backup IED for IEC 61850-based substation. IEEE Transactions on Power Delivery, 28(4): 2048-2055. https://doi.org/10.1109/TPWRD.2013.2258686 